\title{
Commercializing optomechanical sensors: from the classical to quantum regime
}

\author{
Li, Ying Lia, Barker, Peter
}

Ying Lia Li, Peter F. Barker, "Commercializing optomechanical sensors: from the classical to quantum regime," Proc. SPIE 11083, Optical Trapping and Optical Micromanipulation XVI, 110830X (9 September 2019); doi: 10.1117/12.2530147

Event: SPIE Nanoscience + Engineering, 2019, San Diego, California, United States 


\title{
Commercializing optomechanical sensors: from the classical to quantum regime
}

\author{
Ying Lia Li and Peter F. Barker \\ University College London, Gower Street, London WC1E 6BT, United Kingdom;
}

\begin{abstract}
Cavity optomechanical systems show great promise as force and displacement sensors, with scope to operate across the classical to quantum regimes. I will discuss the commercial development of an optical whispering gallery mode (WGM) accelerometer, which relies on a dispersive and dissipative coupling between the cavity resonance and the motion of the cavity. The accelerometer operates at a sensitivity of micro- $\mathrm{g} \mathrm{Hz}^{-1 / 2}\left(\mathrm{~g}=9.81 \mathrm{~ms}^{-2}\right)$ with plans to approach nano-g $\mathrm{Hz}^{-1 / 2}$ through tailoring the mechanical and optical properties. I also describe the first prototype assembly, results from outdoor field-trials, and recent work using micro-electro-mechanical systems engineering to produce a chip-scale device.
\end{abstract}

Keywords: optomechanics, MEMS, silicon photonics, prototype, accelerometer, optical sensors, field trial, whispering gallery mode, microresonators, waveguides, metrology

\section{INTRODUCTION}

Optomechanical sensors are promising candidates for commercialisation, with many systems demonstrating a sensitivity to displacement surpassing current standards. These systems utilise a coupling between mechanical motion and light, which is resonantly enhanced with an optical cavity such as a Fabry-Perot cavity, ${ }^{1,2}$ spherical cavities that support whispering gallery modes (WGMs), ${ }^{3-11}$ and photonic crystal structures. ${ }^{12,13}$ Accelerometers and gyroscopes are created if the mechanical element displaces in response to inertial forces. A dispersive and/or dissipative coupling causes the optical resonance frequency to shift and/or broaden in linewidth respectively. This provides higher signal-to-noise when compared with non-cavity enhanced schemes such as monitoring the amount of light coupled into a movable optical fiber test-mass. ${ }^{14}$ With suitable test-mass engineering to increase the motional response, for example, attaching the optical cavity to a cantilever or pendulum, extremely small accelerations or rotations can be detected when there are high optomechanical coupling rates.

The most commercially successful type of inertial sensor, used widely across all markets, are micro-electromechanical systems (MEMS) sensors which measure the capacitance change between a fixed electrode and a movable one. ${ }^{15}$ Due to their low cost $(\ll \$ 1 /$ chip), MEMS sensors are found within smartphones, drones, biotracking hardware and navigation devices. To compete, bulky optomechanical experiments must be prototyped and miniaturised. This is now an active research area, where MEMS processes are used to create chip-scale silicon photonic structures on silicon-on-insulator wafers, resulting in sub-milimeter scale Fabry-Perot cavities, ${ }^{16}$ evanescent strip waveguides replacing tapered optical fibers, ${ }^{17}$ tethered photonic crystal membranes,${ }^{18}$ and suspended strings with ultrahigh mechanical quality factors. ${ }^{19}$ The significant size reduction offered by optomechanical MEMS sensors, combined with their ability to reach displacement sensitivities on the order of $10^{-18} \mathrm{mHz}^{-1 / 2},{ }^{7,20}$ makes them a particularly disruptive technology. Many optomechanical systems, including the one reported in this proceedings, were originally developed to probe macroscopic quantum behavior by cooling the centre-of-mass motion of the test-mass towards an average phonon occupancy $<1 .{ }^{21}$ Some systems have been cooled to the ground state ${ }^{22}$ and can approach fundamental limits set by quantum mechanics, ${ }^{7}$ which paves the way towards sensing below the zero-point motion via mechanical squeezing ${ }^{23}$ or using a quantum superposition of position to detect weak gravitational forces. ${ }^{24}$

In this proceedings paper we present an optomechanical accelerometer comprising of a spherical silica microcavity supporting optical whispering gallery mode (WGM) resonances, attached to a silica cantilever to form a

Further author information: (Send correspondence to Y.L.Li)

Y.L.Li.: E-mail: ucaplic@ucl.ac.uk

Optical Trapping and Optical Micromanipulation XVI, edited by Kishan Dholakia,

Gabriel C. Spalding, Proc. of SPIE Vol. 11083, 110830X · (c) 2019 SPIE

CCC code: $0277-786 \mathrm{X} / 19 / \$ 21 \cdot$ doi: $10.1117 / 12.2530147$

Proc. of SPIE Vol. $11083110830 \mathrm{X}-1$

Downloaded From: https://www.spiedigitallibrary.org/conference-proceedings-of-spie on 22 Jun 2020 
'microsphere-cantilever' test-mass. A waveguide is used to evanescently couple light to the WGM. During applied acceleration, the microsphere-cantilever deflects, altering the evanescent coupling, which changes the WGM resonance through a dispersive and dissipative coupling. Unlike other WGM accelerometer schemes which detect an external test-mass placed within the WGM's evanescent field, ${ }^{8,9}$ we use the WGM cavity as the test-mass itself. A hand-assembled WGM accelerometer prototype with a sensitivity of $40 \mu \mathrm{g} \mathrm{Hz}$ which survives shocks of $\pm 60 \mathrm{~g}$ during an outdoor field-trial. ${ }^{25}$ Lastly, progress towards the development of a chip-scale MEMS WGM accelerometer is reported.

\section{TRANSDUCTION OF ACCELERATION}

At the heart of the WGM accelerometer is a dispersive and dissipative optomechanical coupling between the WGM to displacement; in this case, the displacement of the WGM cavity itself. This is described in detail in our previous paper ${ }^{26}$ with a summary presented here. To excite WGMs within silica microspheres (or within any dielectric medium with a curved boundary), an evanescent waveguide is used for coupling photons into and out of the cavity. ${ }^{27}$ There are three main coupling rates that define this photon exchange, shown in Fig. 1 (a); the extrinsic coupling $\kappa_{\mathrm{e}}$ which defines light transfer from waveguide to WGM, the intrinsic coupling $\kappa_{\mathrm{i}}$, and a scattering component $\kappa_{\mathrm{s}}$. The material and surface quality of the resonator limits $\kappa_{\mathrm{i}}$, whereas $\kappa_{\mathrm{s}}$ accounts for optical losses that do not couple back into the waveguide. ${ }^{6} \kappa_{\mathrm{e}}$ and $\kappa_{\mathrm{s}}$ have an exponential dependence due to the decay length of the evanescent field of the waveguide and sphere, $\alpha$, such that $\kappa_{\mathrm{e}}=\kappa_{\mathrm{e}, \mathrm{d}=0} e^{-\alpha d}$, and $\kappa_{\mathrm{s}}=\kappa_{\mathrm{s}, \mathrm{d}=0} e^{-\alpha d}$. If $d$ changes, $\kappa_{\mathrm{e}}$ and $\kappa_{\mathrm{s}}$ cause the WGM linewidth to broaden/narrow, known as a dissipative

(a)

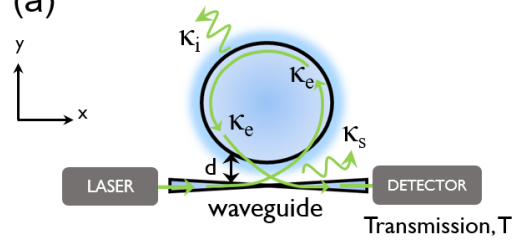

(b)

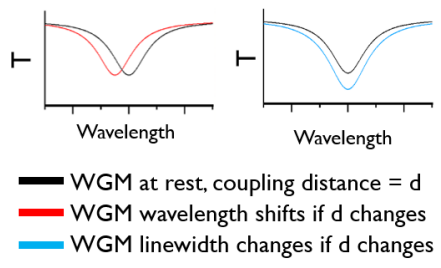

(c)

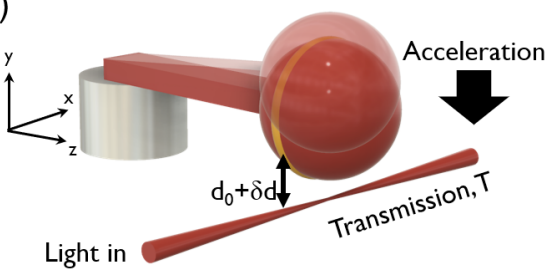

Figure 1. (a) Optical coupling rates $\kappa_{\mathrm{e}}, \kappa_{\mathrm{i}}$, and $\kappa_{\mathrm{s}}$ define photon exchange between an evanescent waveguide and a WGM cavity at a particular coupling distance, $d$. (b) If $d$ changes, the WGM shifts in resonant frequency and broadens/narrows in linewidth. (c) If the WGM cavity is placed on a cantilever, it will displace a distance $\delta d$ proportional to the applied acceleration, which can be transduced by the WGM and measured in the transmission.

coupling. The WGM also shifts in resonant frequency due to a dispersive coupling such that the resonance at $d$ is $\omega_{0}^{\prime}=\omega_{0}-\omega_{0, \mathrm{~d}=0} e^{-\alpha d}$, where $\omega_{0}$ is the resonance frequency when the WGM is barely coupled. Both effects are displayed in Fig. 1 (b). Since the cavity photon lifetime is many orders of magnitude shorter than the acceleration timescales to be considered, the steady-state condition is applied such that the change in photon number is $\frac{\mathrm{d} a}{\mathrm{~d} t}=0$. The normalized transmission can therefore be expressed as $T=\left|\frac{a_{\text {out }}}{a_{\text {in }}}\right|^{2}$, where $a_{\text {in }}$ and $a_{\text {out }}$ are photons leaving and entering the cavity:

$$
T=\left|1-\frac{\kappa_{\mathrm{e}}}{\frac{\kappa_{\mathrm{i}}}{2}+\frac{\kappa_{\mathrm{e}}}{2}+\frac{\kappa_{\mathrm{s}}}{2}+i \Delta}\right|^{2} .
$$

One can create an accelerometer by placing the cavity at an equilibrium coupling point, $d_{0}$, and detecting small displacements $\delta d$ about that equilibrium point. To do this, we place the WGM cavity onto a cantilever which responds to applied forces via Hooke's Law and Newton's 2nd law of motion. An acceleration, $A(t)$, in the vertical direction causes the cantilever to deflect by a distance $\delta d(t)$, as illustrated in Fig. $1(\mathrm{c})$.

$$
A(t)=-\frac{k}{m_{\mathrm{eff}}} \delta d(t)=-\Omega_{\mathrm{m}}^{2} \delta d(t)
$$


where $\Omega_{\mathrm{m}}$ is the fundamental mechanical resonance frequency of the microsphere-cantilever, the spring constant is $k$ and the effective mass is $m_{\text {eff }}$. We approximate the response of the WGM using a linear scale-factor by only considering small motions. This means that the optomechanical coupling rates, which describe the magnitude of change to the WGM per meter of motion, are derived by $\gamma_{\mathrm{om}}\left(d_{0}\right)=\frac{\mathrm{d} \kappa_{\mathrm{e}}\left(d_{0}\right)}{\mathrm{d} d_{0}}($ dissipative $), \gamma_{\mathrm{s}}\left(d_{0}\right)=\frac{\mathrm{d} \kappa_{\mathrm{s}}\left(d_{0}\right)}{\mathrm{d} d_{0}}$ (scattering), and $g_{\mathrm{om}}\left(d_{0}\right)=\frac{\mathrm{d} \Delta\left(d_{0}\right)}{\mathrm{d} d_{0}}$ (dispersive). These linearised optomechanical rates work together to alter $T(t)$ when acceleration causes $\delta d(t)$ as so: ${ }^{12,26}$

$$
\mathrm{d} T(t)=\left|g_{\mathrm{om}} \frac{\partial T}{\partial \Delta}+\gamma_{\mathrm{om}} \frac{\partial T}{\partial \kappa_{\mathrm{e}}}+\gamma_{\mathrm{s}} \frac{\partial T}{\partial \kappa_{\mathrm{s}}}\right| \delta d(t) .
$$

The derivatives are shown in Appendix A. The ratio between $g_{\mathrm{om}}, \gamma_{\mathrm{om}}$, and $\gamma_{\mathrm{s}}$ defines the scale-factor at each $d_{0}$. From our previous studies, the magnitude of the optomechanical coupling rates are around $\mathrm{MHz} / \mathrm{nm}$, with the most sensitive WGM accelerometer obtaining a noise density of $4 \mu \mathrm{g} \mathrm{Hz}{ }^{1 / 2}$, limited by classical detection noise, and a scale-factor of $15 \mathrm{~V} / \mathrm{g}\left(\mathrm{g}=9.81 \mathrm{~ms}^{-2}\right)$ when using an amplified photodetector. ${ }^{26}$

\section{A PROTOTYPE WHISPERING GALLERY MODE ACCELEROMETER}

To test the feasibility of a WGM accelerometer used outside of the laboratory environment, a portable battery powered prototype was assembled. This was field-tested on a military vehicle akin to the Jackal ${ }^{28}$ and was shown to function and survive when subjected to shocks of $\pm 60 \mathrm{~g}$. Full details of the set-up with analysis of the field-test can be found in our prototype paper. ${ }^{25}$ Here we present an overview of the prototype, summarise what we learnt from the field-trial, and highlight unpublished trial data showing functionality as a vibration sensor capable of differentiating between different terrains.

\subsection{Prototype overview}

This early stage prototype is completely hand-assembled and is a good example of accessible, rapid prototyping. Costing $£ 30 \mathrm{k}$ to build within 1 year, it demonstrates what can be achieved without significant funding or resources; no clean-room fabrication or low vacuum pressures are required. The core of the prototype is the sensor head, shown in Fig. 2, comprising of the microsphere-cantilever and the evanescent waveguide. $\mathrm{A} \mathrm{CO}_{2}$ laser is used to melt the tip of a rectangular core fiber $(240 \mu \mathrm{m} \times 106 \mu \mathrm{m})$ to form a microsphere $(350 \mu \mathrm{m}$ diameter) that remains attached to a cantilever stem (2.2 $\mathrm{mm}$ length). A butane torch and two motors are used to taper an optical fiber by melting it whilst pulling in either direction, creating an evanescent waveguide epoxied onto a mount at four positions. To reduce extraneous mechanical degrees of freedom from any additional mounts
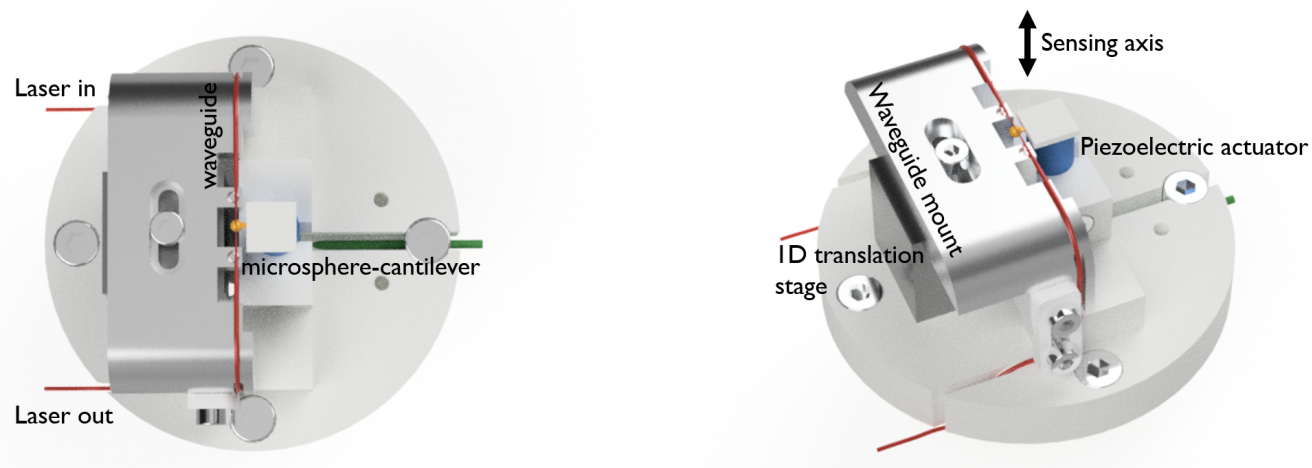

Figure 2. Two views of the sensor head comprising of the microsphere-cantilever and evanescent waveguide, aligned with a manual 1-D translation stage and a piezo-electric actuator.

and translation stages, the microsphere-cantilever and waveguide are aligned to an equilibrium coupling distance of $d<1 \mu \mathrm{m}$ using a 1-D manual translation stage and a piezo-electric actuator (PZT). The 1-D stage obtains 
$d<5 \mu \mathrm{m}$ and the PZT allows for fine adjustment to a typical operating distance of $0.5 \mu \mathrm{m}<d<1 \mu \mathrm{m}$. A bespoke base is designed to secure the translation stage and PZT, with guidance channels to secure the optical fiber ends.

The sensor head is placed inside a chamber at atmospheric pressure which is bolted into the prototype package, shown in Fig. 3 (a). Also inside is the tunable $1550 \mathrm{~nm}$ DFB laser source, a National Instruments CompactRIO with built-in FPGA for automating the prototype, and two commercial sensors detailed in Appendix B. A control panel, Fig. 3 (b) distributes power from portable batteries $(+12 \mathrm{~V}$ and $-12 \mathrm{~V}$ supply) and allows communication between the user and prototype via switches and LED indicators. For a full description of the prototype and the FPGA protocols, refer to our previous paper. ${ }^{25}$

(a)

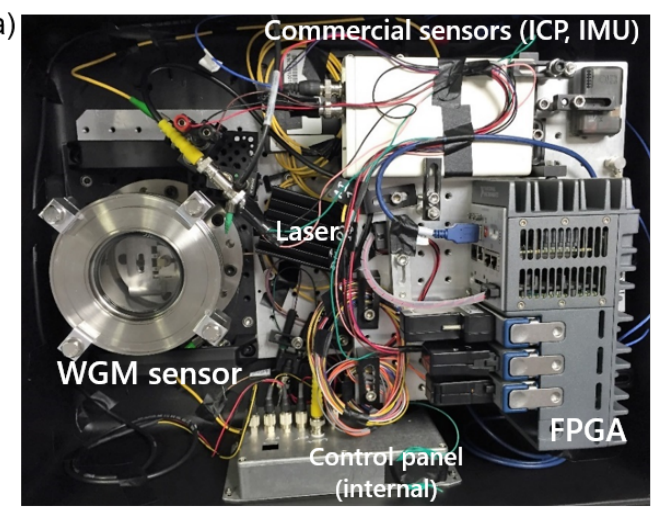

(b)

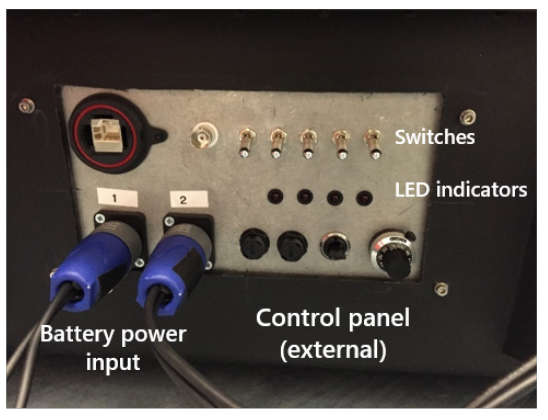

Figure 3. (a) Image of the WGM accelerometer prototype. (b) External view of the control panel.

\subsection{Controlled shaker testing versus field-testing}

The prototype was first tested using an industry standard electrodynamic shaker which applied a fixed frequency sinusoidal acceleration up to $\pm 6 \mathrm{~g}$. For many academic endeavors, conducting a controlled shaker test is sufficient for demonstrating sensor functionality. However, for a commercialisation path where the sensor must be cost effective and robust, field-testing is a valuable tool for tailoring each iteration towards market requirements. For this reason, the prototype underwent a rough test on a military vehicle outdoors, mounted in the direction of travel. Here we summarise the lessons learnt from conducting the controlled shaker test versus the field-trial (sections 3.2.1, 3.2.2 \& 3.2.3 describe previously published data, with unpublished data shown in Figs. $4 \& 5$ ).

\subsubsection{Cross-correlation}

A normalised cross-correlation was performed that compares the output of the WGM prototype to a commercial integrated circuit piezoelectric (ICP) accelerometer which is factory calibrated and shipped with the electrodynamic shaker.

Shaker test: Excellent cross-correlation of $r=0.997$, demonstrating negligible lag or non-linearities in response. $r=1$ would imply the output of both sensors are exactly the same.

Field-trial: Poor cross-correlation of $r=0.36$, which is due to issues to detailed in next two sections.

\subsubsection{Scale-factor linearity}

Shaker test: Highly linear scale-factor, with fitting error $<0.2 \%$. However, the weight of the sensor head within the chamber was nearly $6 \mathrm{~kg}$, severely limiting the test-range to $\pm 6 \mathrm{~g}$. The threshold for causing damage to the prototype and/or the onset of a non-linear response cannot be tested.

Field-trial: Outdoor ambient temperature fluctuations of $\pm 0.2^{\circ} \mathrm{C}$ shift the WGM resonance and distort the scale-factor. When analysing peak acceleration detection, equivalent to measuring the envelope of the time trace, the cross correlation improved to $r=0.92$, which suggests that temperature effects only partially account for errors. Since the vehicle produced extreme shock profiles by driving at $20 \mathrm{mph}$ into barriers/curbs, we were able to demonstrate survival \& functionality during $\pm 60 \mathrm{~g}$ and show that the transduction becomes non-linear at these high accelerations. 


\subsubsection{Sensitivity}

Shaker test: The Allen deviation defined a sensitivity of $40 \mu \mathrm{g} \mathrm{Hz}^{-1 / 2}$ but the power spectral density showed a non-flat noise floor. The sensitivity must therefore be quoted in frequency bands: $40 \mu \mathrm{g} \mathrm{Hz}^{-1 / 2}$ at frequencies between $2-7 \mathrm{kHz}$, increasing to $130 \mu \mathrm{g} \mathrm{Hz}^{-1 / 2}$ at $200 \mathrm{~Hz}$, and $250 \mu \mathrm{g} \mathrm{Hz}^{-1 / 2}$ at $100 \mathrm{~Hz}$. Flicker noise decreases the sensitivity at low frequency. Closed-loop feedback was used to stabilise the equilibrium coupling distance, counteracting unwanted drift and creep of the PZT which alters the coupling distance. Due to the large size of the microsphere-cantilever, stable actuation forces such as optical forces or capacitive actuation cannot be used. This means that slow accelerations associated with navigation cannot be captured, only vibration and shock.

Field-trial: The appearance of a mechanical resonance around $30 \mathrm{~Hz}$ becomes apparent under certain testconditions, which adds false acceleration readings and lowers the cross-correlation. Closed-loop feedback performed well, maintaining the equilibrium coupling distance for durations $>10$ mins long and after large shocks.

It is clear that the hand-fabricated nature of the prototype degrades its operation in real-life scenarios. Temperature stabilisation is not easily achieved with bulky structures and additional and unwanted mechanical responses from mounts distort the scale-factor at certain frequencies, or become resonant under certain conditions. It should be noted that these errors are not associated with the fundamental transduction mechanism. Packaging and mounting position within the vehicle can create discrepancies between different sensors, for example, when comparing the cross-correlation and power spectral density of an accelerometer from an inertial measurement unit (IMU, specifications in Appendix B) to the ICP test-standard, one finds similar issues: a low $r=0.33$ and non-linear responses at certain frequencies, shown in Figs. 4 (a), (b), \& (c). Also shown are the time trace, cross-correlation, and power spectral density of the WGM response.
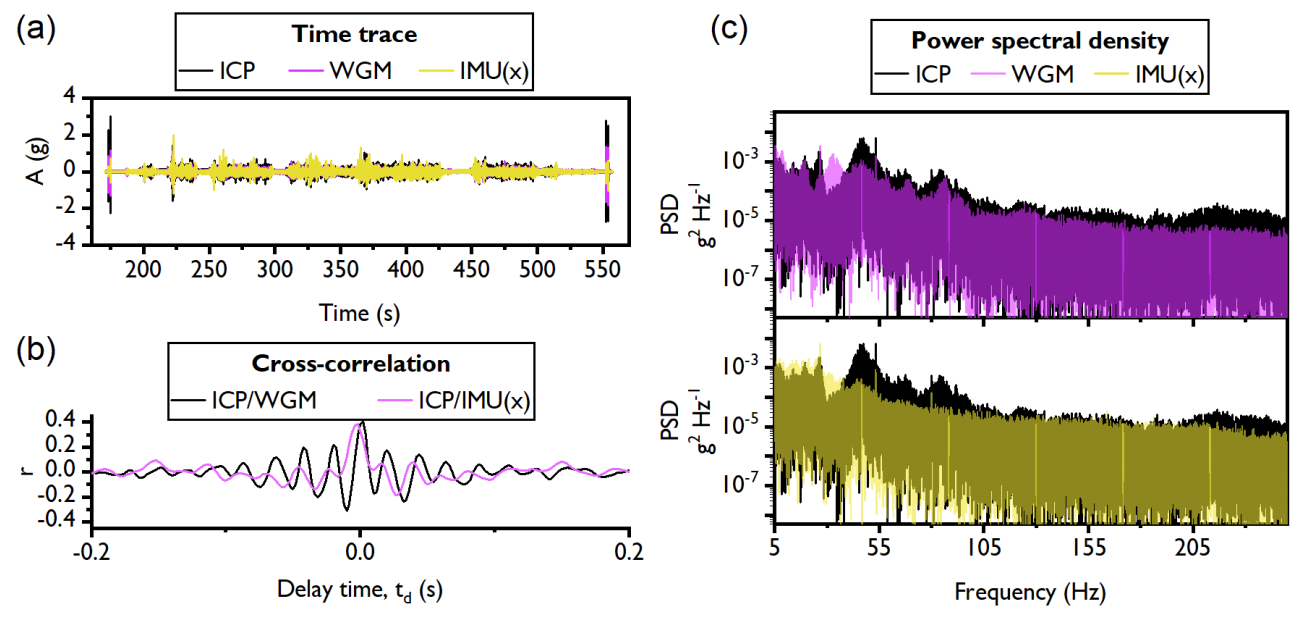

Figure 4. (a) Time trace of the responses from the WGM accelerometer prototype and two commercial sensors during a field-trial. (b) Cross-correlation as a function of time delay between the ICP sensor with the WGM and IMU (x-axis accelerometer output) sensors. (c) The power spectral density (PSD) of the WGM and IMU(x) accelerometers overlayed onto the ICP test-standard.

Attempts by others to correlate the signals from different brands of accelerometers on vehicles results in similar levels of moderate to weak cross-correlation, for example, $0.02<r<0.65$ is found between the accelerometers in the Samsung Galaxy S5 versus the LSDM202DLM-3 sensor. ${ }^{29}$ Future tests on vibrational platforms like vehicles should investigate this further by testing multiple arrays of sensors.

Taking into account the weaknesses and strengths of this system, the prototype in its current form would still function well as a vibration sensor for impact detection, ${ }^{30}$ or to trigger alerts when transporting goods with a damage threshold. ${ }^{31}$ As an example, we show how the sensor is able to differentiate between different types of vibrations and shocks, and how this can be used to determine the type of terrain. In Fig. 5 are three segments of the trial-data presented as time traces alongside the power spectral density; (a) shows two hammer shocks applied directly to the stationary vehicle, (b) is taken when the vehicle traverses across tarmac, and (c) traverses 
across potholed grassland. The ICP commercial sensor is used as the test-standard for comparison with the WGM accelerometer and the IMU accelerometer.
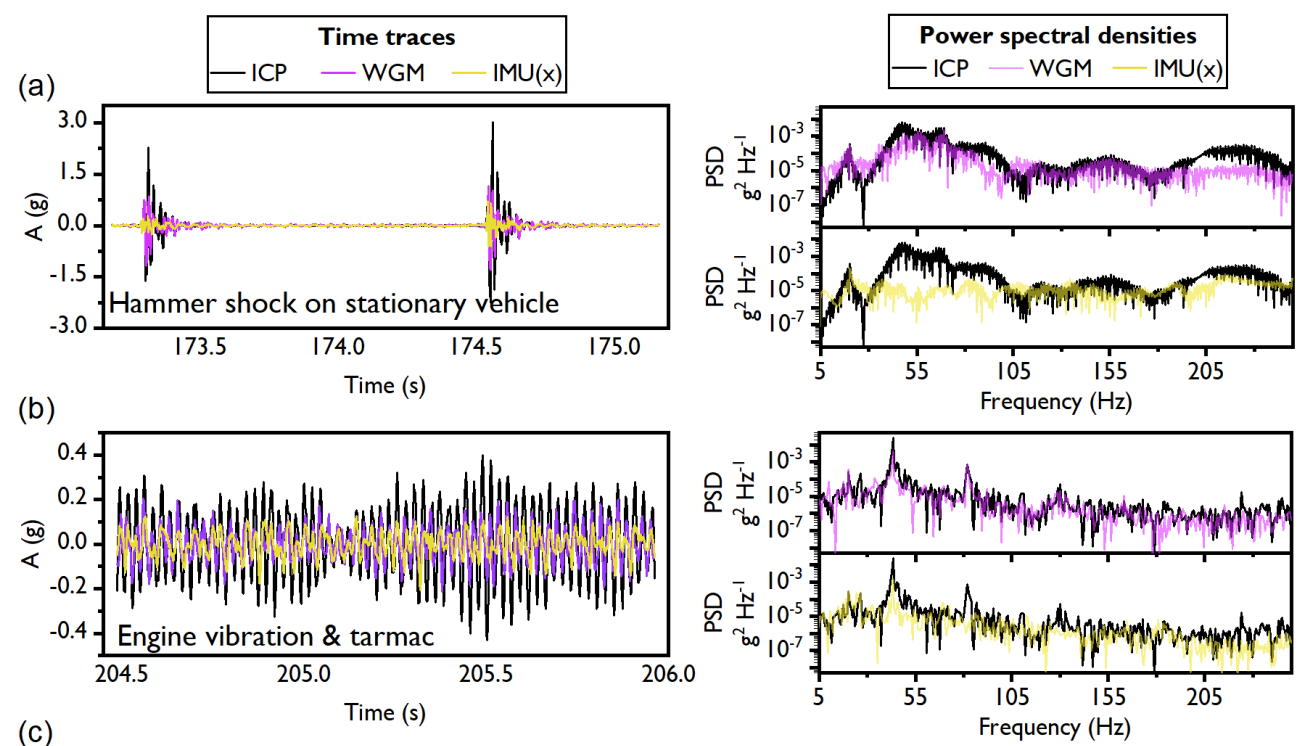

(c)

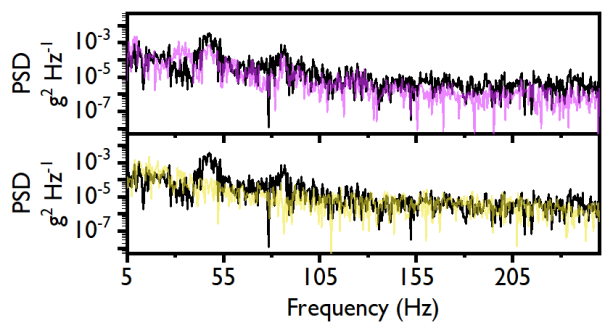

Figure 5. Segments of the trial data from Fig. 4 and the corresponding power spectral densities, comparing the WGM and IMU accelerometers to the ICP sensor: (a) Hammer strikes onto a stationary vehicle with engine off, (b) Moving across tarmac with engine on, (c) Moving across potholed grassland with engine on.

The power spectral density of the WGM accelerometer for the complete trial run (Fig. 4) and the segments in Fig. 5 show good agreement with the ICP sensor. It is consistently better than the IMU response.

The hand-assembled prototype shows great promise as a commercial device but reducing errors and drift cannot be achieved if one continues to hand-assemble the device. To improve and miniturise the WGM accelerometer further, we turn to precision manufacturing, detailed in the next section.

\section{TRANSITION TO CHIP-SCALE OPTOMECHANICS}

Micro-electro-mechanical systems (MEMS) engineering involves deposition, lithography, and etching to create structures on the micro-to-nanoscale. MEMS fabrication is used to create ultrasensitive, low-drift electrical accelerometers and gyroscopes used for navigation, wearable devices, image stabilisation and many more applications. ${ }^{15}$ Optical elements can be MEMS fabricated, with micro-opto-electro-mechanical systems (MOEMS) becoming a rapidly growing field, ${ }^{32}$ benefiting from the high yield and reproducibility of MEMS fabrication. For our sensor, MEMS offers a way to align the waveguide and WGM cavity via chip-bonding, eliminating any use of translation stages, mounts, or piezo-electric actuation.

We report on our progress with developing a chip-scale WGM accelerometer using MEMS techniques. Here we focus on designing and testing the photonic elements only, which comprise of an evanescent waveguide and a WGM cavity. We use a standard silicon-on-insulator (SOI) process, offered by the multi-process wafer service 


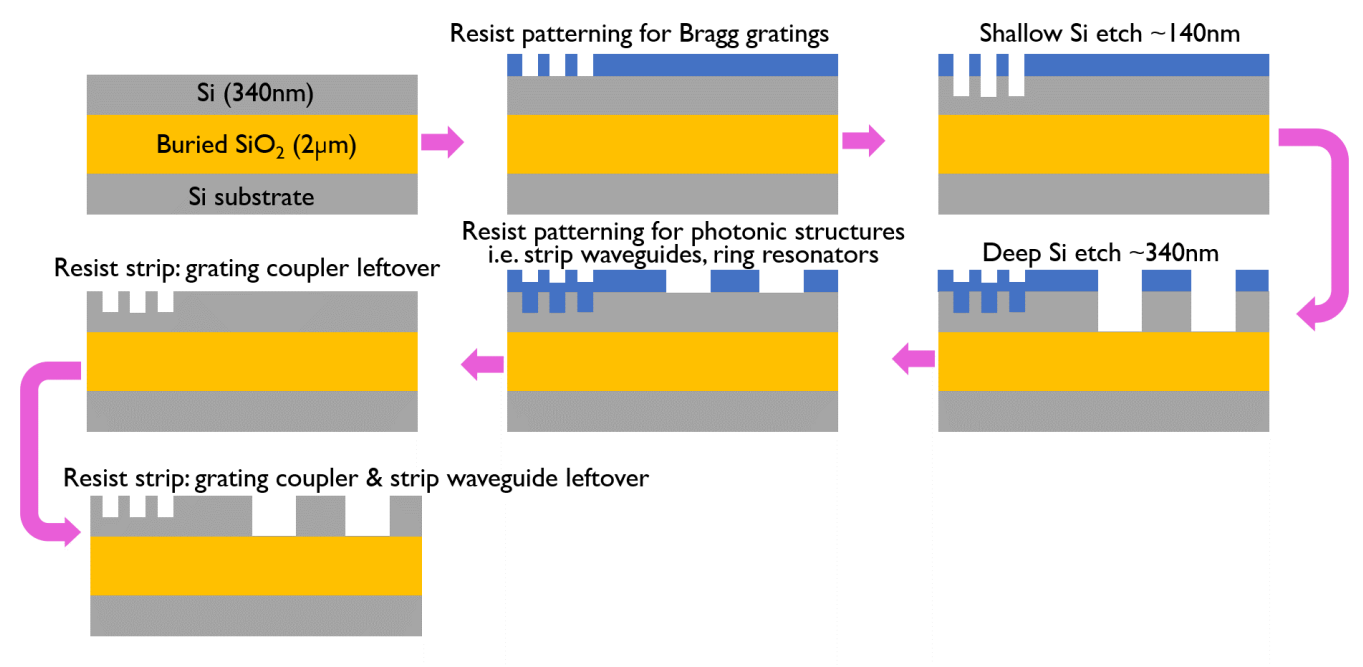

Figure 6. Process flow to create photonic strip waveguides and ring resonators on-chip.

(a)

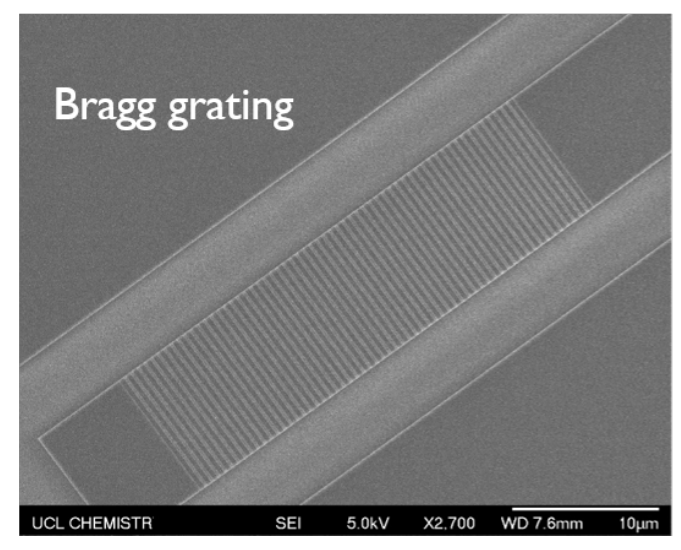

(b)

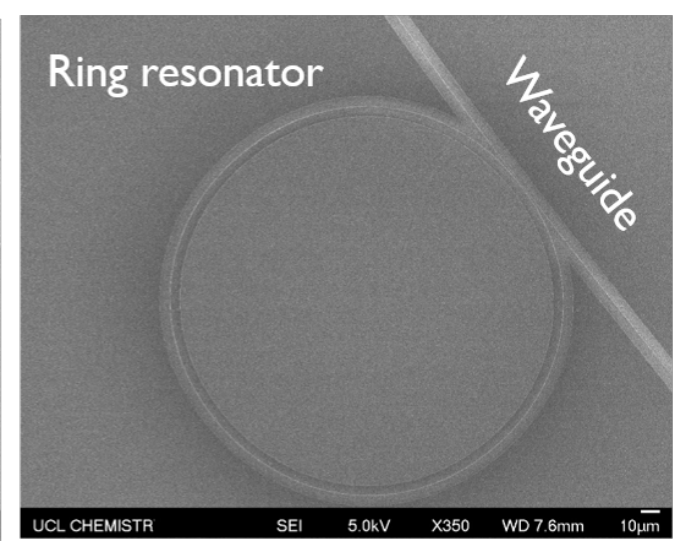

Figure 7. Scanning electron microscope images of photonic elements on chip. (a) Bragg grating attached to a strip waveguide. (b) Ring resonator coupled to a strip waveguide.

from Southampton clean-room CORNERSTONE, outlined in Fig. 6. The tapered optical fiber waveguide used previously is now replaced by a strip waveguide (width $400 \mathrm{~nm}$, depth $340 \mathrm{~nm}$ ). Light is coupled into and out of the strip waveguide via Bragg gratings, as shown in Fig. 7(a). The grating linewidth is $0.325 \mu \mathrm{m}$ (gap=0.265 $\mu \mathrm{m}$ ), and grating depth of $140 \mathrm{~nm}$, with 60 gratings in total. This is optimised for the TE mode at $1550 \mathrm{~nm}$. Also fabricated are WGM cavities in the form of ring resonators, which are positioned at a fixed coupling distance away strip waveguides, in Fig. 7(b). Work towards creating a WGM cavity on top of a cantilever has not yet begun. This is because underetching is required to create a movable, suspended cantilever and requires further investigation.

A chip testing rig was set-up to probe the photonic structures by sending $1550 \mathrm{~nm}$ light through the input Bragg grating of a strip waveguide coupled to a ring resonator, and collecting the transmitted light at the output Bragg grating. The test rig is shown in Fig. 8 (a), with a microscope image of a ring resonator (diameter=160 $\mu \mathrm{m}$ ) in (b), and the output coupling fiber in (c). A tunable $1550 \mathrm{~nm}$ is scanned from $1515 \mathrm{~nm}$ to $1575 \mathrm{~nm}$, revealing a spectrum of WGMs in Fig. 9 (a), with a transmission envelope defined by the Bragg gratings. These WGMs typically have FWHM linewidths of GHz $(3.7 \mathrm{GHz}$ WGM shown in Fig. $9(\mathrm{~b}))$. The optical quality factor of $5.2 \times 10^{4}$ is a few orders of magnitude lower than for the microspheres created by $\mathrm{CO}_{2}$ laser ablation. It should 
(a)

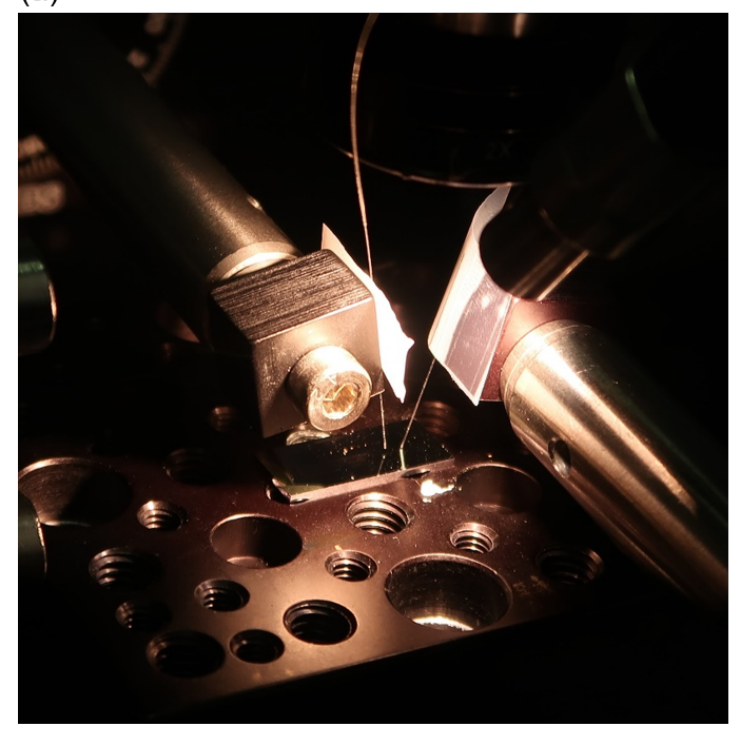

(b)

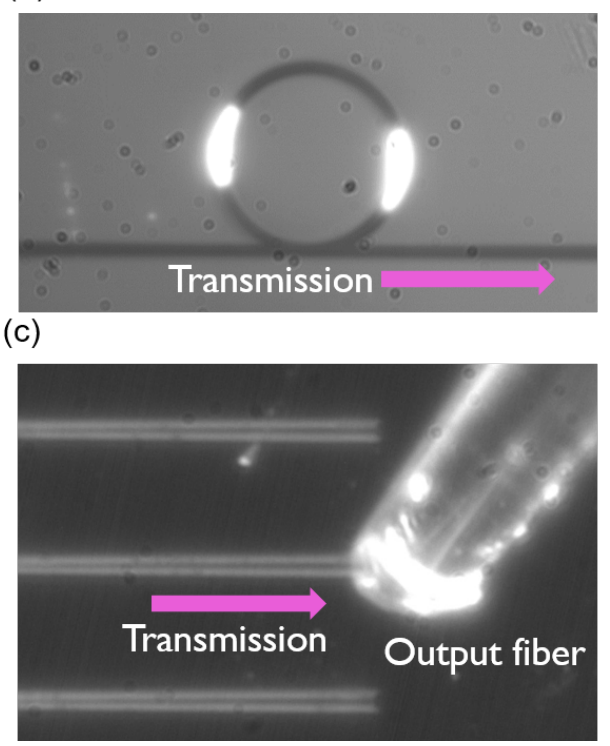

Figure 8. (a) Photonic chip test-rig positioning two stripped optical fiber ends onto the input and output Bragg gratings of a strip waveguide. (b) A ring resonator coupled to a strip waveguide. (c) The output fiber collecting transmitted light through a strip waveguide.

(a)

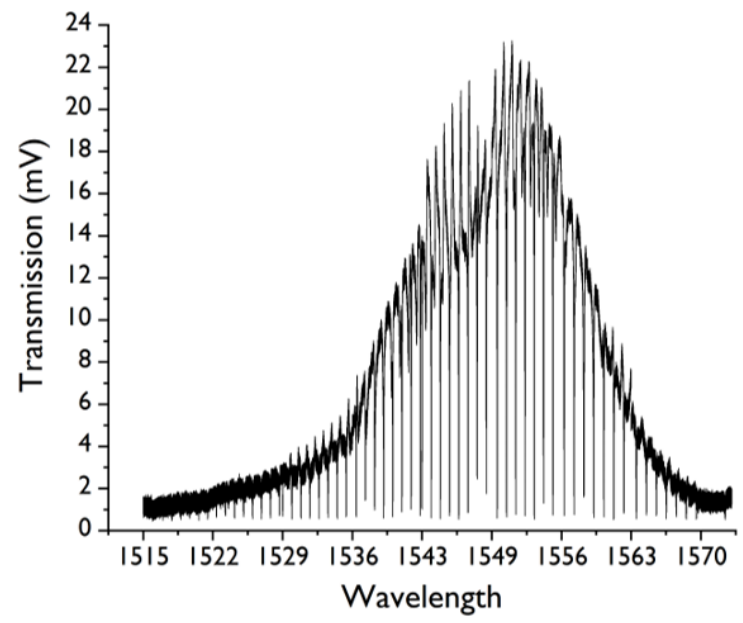

(b)

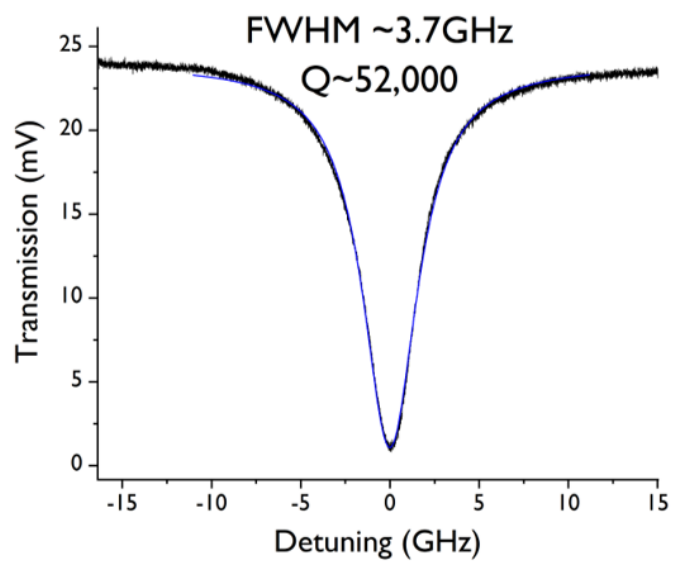

Figure 9. (a) Transmission of a strip waveguide coupled to a ring resonator on-chip when the laser is scanned. (b) Typical WGM profile, with $3.7 \mathrm{GHz}$ FWHM. 
be possible to improve the optical quality factor by creating a smoother surface through optimising the etching process. Others have achieved optical quality factors of $2.4 \times 10^{7}$ in wedge microdisks ${ }^{33}$ and $5 \times 10^{6}$ for microdisks undergoing a post-lithography bake to reduce imperfections in the resist patterning. ${ }^{34}$

The last stage of hardware development will create a WGM resonator on a cantilever. A microdisk style cavity will be used, attached to a cantilever whose thermomechanical motion is sufficiently low to reach sensitivities approaching $\mathrm{n} \mathrm{Hz}^{-1 / 2}$. An exciting possibility for future work is cooling the microdisk-cantilever structure to its centre-of-mass ground state. We previously demonstrated centre-of-mass cooling with the hand-assembled microsphere-cantilevers but the cooling was limited by the poor mechanical properties of the cantilever and detection noise. ${ }^{3}$ MEMS engineering can be used to create optimised cantilevers that can be used for sensing beyond classical limits when cooled to the quantum ground state.

\section{CONCLUSION}

We report on the commercialisation path of a whispering gallery mode (WGM) accelerometer which transduces acceleration when the WGM cavity is attached to a cantilever. This system has been tested in the lab, ${ }^{26}$ transformed into a prototype undergoing outdoor field-testing, ${ }^{25}$ and is now under development in chip-form using MEMS fabrication.

\section{APPENDIX A. SCALE-FACTOR DERIVATIVES}

For the WGM system studied here;

$$
\begin{gathered}
\frac{\partial T}{\partial \kappa_{\mathrm{e}}}=-\frac{4\left(\kappa_{\mathrm{i}}+\kappa_{\mathrm{s}}\right)\left(4 \Delta^{2}-\kappa_{\mathrm{e}}^{2}+\left(\kappa_{\mathrm{i}}+\kappa_{\mathrm{s}}\right)^{2}\right)}{\left(4 \Delta^{2}+\kappa^{2}\right)^{2}}, \\
\frac{\partial T}{\partial \Delta}=\frac{32 \Delta \kappa_{\mathrm{e}}\left(\kappa_{\mathrm{i}}+\kappa_{\mathrm{s}}\right)}{\left(4 \Delta^{2}+\kappa^{2}\right)^{2}}, \text { and } \\
\frac{\partial T}{\partial \kappa_{\mathrm{s}}}=-\frac{4 \kappa_{\mathrm{e}}\left(4 \Delta^{2}+\kappa_{\mathrm{e}}^{2}-\left(\kappa_{\mathrm{i}}+\kappa_{\mathrm{s}}\right)^{2}\right)}{\left(4 \Delta^{2}+\kappa^{2}\right)^{2}} .
\end{gathered}
$$

\section{APPENDIX B. COMMERCIAL SENSOR SPECIFICATIONS}

\begin{tabular}{l|l|l} 
Sensor & Best sensitivity, $\mu \mathrm{g} \mathrm{Hz}^{-1 / 2}$ & Model \\
\hline WGM & 40 & Prototype \\
ICP & 3.4 & 352 C33 by PCB Piezotronics \\
IMU & 218 & x-IMU by x-io (LSM303DLH accelerometers)
\end{tabular}

Table 1. Specification of the WGM accelerometer and two commercial sensors deployed in the field-trial.

For the field-trial analysis within this proceedings paper, all three sensor outputs are interpolated to a common sampling rate of $500 \mathrm{~Hz}$ with a $250 \mathrm{~Hz} \mathrm{LPF}$ and $5 \mathrm{~Hz}$ HPF applied to the ICP and WGM signals.

\section{ACKNOWLEDGMENTS}

The authors would like to thank CORNERSTONE and Dr. Callum Littlejohns for fabricating the photonic chips in Fig. 7, Markus Rademacher for taking the SEM images of the chip structures in Fig. 7, and Zhimeng Wu for helping to assemble the chip test-rig. 


\section{REFERENCES}

[1] O. Gerberding, F. G. Cervantes, J. Melcher, J. R. Pratt, and J. M. Taylor, "Optomechanical reference accelerometer," Metrologia, vol. 52, no. 5, pp. 654-665, Sep. (2015).

[2] LIGO Scientific Collaboration \& Virgo Collaboration, "Observation of gravitational waves from a binary black hole merger," Phys. Rev. Lett., vol. 116, p. 061102, Feb. (2016).

[3] Y. L. Li, J. Millen, and P. F. Barker, "Simultaneous cooling of coupled mechanical oscillators using whispering gallery mode resonances," Optics Express, vol. 24, no. 2, pp. 1392-1401, Jan. (2016).

[4] M. Li, W. H. P. Pernice, and H. X. Tang, "Reactive cavity optical force on microdisk-coupled nanomechanical beam waveguides," Phys. Rev. Lett., vol. 103, p. 223901, Nov. (2009).

[5] M. Eichenfield, C. P. Michael, R. Perahia, and O. Painter, "Actuation of micro-optomechanical systems via cavity-enhanced optical dipole forces," Nature Photonics, vol. 1, pp. 416-422, Jul. (2007).

[6] R. Madugani, Y. Yang, J. M. Ward, V. H. Le, and S. Nic Chormaic, "Optomechanical transduction and characterization of a silica microsphere pendulum via evanescent light," Appl. Phys. Lett., vol. 106, p. 241101, Dec. (2015).

[7] A. Schliesser, O. Arcizet, R. Riviére, G. Anetsberger, and T. J. Kippenberg, "Resolved-sideband cooling and position measurement of a micromechanical oscillator close to the Heisenberg uncertainty limit," Nature Physics, vol. 5, pp. 509-514, Jun. (2009).

[8] H. Miao, K. Srinivasan, and V. Aksyuk, "A microelectromechanically controlled cavity optomechanical sensing system," New Journal of Physics, vol. 14, p. 075015, Jul. (2012).

[9] E. Gavartin, P. Verlot, and T. J. Kippenberg, "A hybrid on-chip optomechanical transducer for ultrasensitive force measurements," Nature Nanotechnology, vol. 7, pp. 509-514, Jun. (2012).

[10] G. Anetsberger, E. M. Weig, J. P. Kotthaus, and T. J. Kippenberg, "Cavity optomechanics and cooling nanomechanical oscillators using microresonator enhanced evanescent near-field coupling," Comptes Rendus Physique, vol. 12, no. 9-10, pp. 800-816, Dec. (2011).

[11] J.-P. Laine, C. Tapalian, B. Little, and H. Haus, "Acceleration sensor based on high-Q optical microsphere resonator and pedestal antiresonant reflecting waveguide coupler," Sensors and Actuators A, vol. 93, no. 1, pp. 1-7, Aug. (2001).

[12] M. Wu, A. C. Hryciw, C. Healey, D. P. Lake, H. Jayajumar, M. R. Freeman, J. P. Davis, and P. E. Barclay, "Dissipative and dispersive optomechanics in a nanocavity torque sensor," Phys. Rev. X, vol. 4, p. 021052, Jun. (2014).

[13] A. G. Krause, M. Winger, T. D. Blasius, Q. Lin, and O. Painter, "A high-resolution microchip optomechanical accelerometer," Nature Photonics, vol. 6, pp. 768-772, Mar. (2012).

[14] J. Kalenik and R. Pajak, "A cantilever optical-fiber accelerometer," Sensors and Actuators A: Physical, vol. 68 , no. 1-3, pp. 350-355, Jun. (1998).

[15] D. K. Shaeffer, "MEMS inertial sensors: A tutorial overview," IEEE Communications Magazine, vol. 51, no. 4, pp. 100-109, Apr. (2013).

[16] G. Wachter, S. Kuhn, S. Minniberger, C. Salter, P. Asenbaum, J. Millen, M. Schneider, J. Schalko, U. Schmid, A. Felgner, D. Hüser, M. Arndt, and M. Trupke, "Silicon microcavity arrays with open access and a finesse of half a million," Light: Science and Applications, vol. 8, no. 37, (2019).

[17] M. Ghulinyan, R. Guider, G. Pucker, and L. Pavesi, "Monolithic Whispering-Gallery Mode Resonators With Vertically Coupled Integrated Bus Waveguides," IEEE Photonics Technology Letters, vol. 23, no. 16, pp. 1166-1168, May. (2011).

[18] R. A. Norte, J. P. Moura, and S. Gröblacher, "Mechanical Resonators for Quantum Optomechanics Experiments at Room Temperature," Physical Review Letters, vol. 116, no. 147202, Apr. (2016).

[19] S. A. Fedorov, V. Sudhir, R. Schilling, H. Schütz, D. J. Wilson, and T. J.Kippenberg, "Evidence for structural damping in a high-stress silicon nitride nanobeam and its implications for quantum optomechanics," Physics Letters A, vol. 382, no. 33, pp. 2251-2255, Aug. (2018).

[20] O. Arcizet, P. F. Cohadon, T. Briant, M. Pinard, A. Heidmann, J. M. Mackowski, C. Michel, L. Pinard, O. Français, and L. Rousseau, "High sensitivity optical monitoring of a micromechanical resonator with a quantum limited optomechanical sensor," Phys. Rev. Lett., vol. 97, p. 133601, Sep. (2006). 
[21] M. Aspelmeyer, T. J. Kippenberg, and F. Marquardt, "Cavity Optomechanics," Reviews of Modern Physics, vol. 86, no. 4, pp. 1391-1452, Dec. (2014).

[22] J. D. Teufel, T. Donner, D. Li, J. W. Harlow, M. S. Allman, K. Cicak, A. J. Sirois, J. D. Whittaker, K. W. Lehnert, and R. W. Simmonds, "Sideband cooling of micromechanical motion to the quantum ground state," Nature, vol. 475, pp. 359-363, Jul. (2011).

[23] E. E. Wollman, C. U. Lei, A. J. Weinstein, J. Suh, A. Kronwald, F. Marquardt, A. A. Clerk, and K. C. Schwab, "Quantum squeezing of motion in a mechanical resonator," Science, vol. 349, no. 6251, Aug. (2015).

[24] S. Qvarfort, A. Serafini, P. F. Barker, and S. Bose, "Gravimetry through non-linear optomechanics," Nature Communications, vol. 9, no. 3690, (2018).

[25] Y. L. Li, and P. F. Barker, "Field Evaluation of a Portable Whispering Gallery Mode Accelerometer," Sensors, vol. 18, no. 12, 4184 (2018).

[26] Y. L. Li, and P. F. Barker, "Characterization and Testing of a Micro-g Whispering Gallery Mode Optomechanical Accelerometer," Journal of Lightwave Technology, vol. 36, no. 18, pp. 3919-3926 (2018).

[27] M. Cai, O. Painter, and K. J. Vahala, "Observation of critical coupling in a fiber taper to a silicamicrosphere whispering-gallery mode system," Phys. Rev. Lett., vol. 85, no. 74, pp. 74-77, Jul. (2000).

[28] Supacat product HMT 400. Available online: https://supacat.com/products/hmt/hmt400/

[29] J. P. Ehsani, F. O'Brien, and B. Simons-Morton, Comparing g-force measurement between a smartphone app and an in-vehicle accelerometer. Proceedings of the Ninth International Driving Symposium on Human Factors in Driver Assessment, Training and Vehicle Design, Manchester Village Vermont, United States, June 26-29, (2017), doi:10.17077/drivingassessment.1639

[30] SM. M. Duma, S. J. Manoogian, W. R. Bussone, P. G. Brolinson, M. W. Goforth, J. J. Donnenwerth, R. M. Greenwald, J. J. Chu, J. J. Crisco, Analysis of real-time head accelerations in collegiate football players. Clinical Journal of Sport Medicine, vol. 15, no. 1, pp. 3-8 (2005).

[31] K. Vursavus, and F. Ozgoven, Determining the effects of vibration parameters and packaging method on mechanical damage in golden delicious apples. Turkish Journal of Agriculture and Forestry, vol. 28, pp. 311-320, (2004).

[32] P. R. Choudhury. MEMS and MOEMS Technology and Applications, SPIE Press, ISBN-13: 9780819437167, (2000).

[33] G. Li, P. Liu, X. Jiang, C. Yang, J. Ma, H. Wu, and M. Xiao, High-Q silica microdisk optical resonators with large wedge angles on a silicon chip, Photonics Research, vol. 3, no. 5, pp. 279-282, (2015).

[34] M. Borselli, T. J. Johnson, and O. Painter, Beyond the Rayleigh scattering limit in high-Q silicon microdisks: theory and experiment, Optics Express, vol. 13, no. 5, pp. 1515-1530, (2005). 